\title{
VIEWPOINT
}

\section{Non-cardiac surgery in the heart failure patient}

\author{
Joel P Reginelli, Roger M Mills
}

In this era of increasingly procedural cardiology, many physicians regard the cognitive exercise of preoperative evaluation for non-cardiac surgery as an onerous task. In reality, the preoperative period affords a window of opportunity for the consulting cardiologist to minimise surgical risk by maximising medical treatment, directing appropriate testing, and communicating effectively with the surgical team. Many physicians are unaware that nearly half of all perioperative deaths are related to cardiac events, and they may greatly underestimate the degree to which heart failure contributes to this cardiac mortality. It is imperative as a consulting cardiologist to develop a consistent approach to heart failure patients in order to avoid unnecessary, and potentially dangerous, preoperative diagnostic and therapeutic interventions.

In this article we will briefly review recent data relating to predictors of surgical risks, and propose an approach for integrating the clinical evaluation of cardiac status and surgical risk in order to provide a frame of reference for preoperative consultation. We have no hard evidence, either observational or in the form of prospective, randomised trials, to support many of our current recommendations. The landmark studies of preoperative risk assessment focused primarily on identifying ischemic risk with a lesser emphasis on heart failure. ${ }^{12}$ Our suggestions are based on heart failure data extrapolated from these studies, as well as from broad experience at a high volume cardiac centre.

\section{Determinants of risk}

There are four major determinants of risk for any cardiac patient undergoing non-cardiac surgery: urgency of the surgery; complexity of the procedure; extent of medical comorbidities; and the extent and severity of the underlying heart disease. Shah and associates underscored the importance of these variables in their evaluation of 688 patients with cardiovascular disease undergoing non-cardiac surgery. ${ }^{3}$ Patients requiring emergency surgery had an $18 \%$ incidence of perioperative myocardial infarction or death, as opposed to a $3.7 \%$ incidence in those patients undergoing elective surgery. Similarly, high risk surgical procedures involving intrathoracic, intra-abdominal, or major vascular reconstruction carried an $8.2 \%$ incidence of perioperative myocardial infarction or death as opposed to a $3.5 \%$ incidence of these complications in less physiologically stressful surgery.
In an attempt to dissect medical versus surgical risk factors, Ali and associates assigned patients both a surgical and a medical risk score based on the complexity of the proposed surgery and the patient's cardiac status, respectively. ${ }^{4}$ The medical score provided significantly better risk prediction for adverse perioperative cardiac events than the surgical score. A high medical score identified $87 \%$ of those who suffered a cardiac event, while a high surgical risk score identified only $33 \%$. The authors emphasised that the extent of cardiac disease is an even greater determinant of outcome than the nature of the proposed surgery.

These findings related primarily to ischaemic complications, and did not specifically address complications caused by heart failure. In a later modification of the original Goldman risk assessment, Detsky and colleagues differentiated risk based on recent clinical status. ${ }^{2}$ In this analysis, pulmonary oedema within a week before surgery conferred a substantially higher risk than remote episodes of decompensation.

Once the surgical and medical risk has been assessed, the consultant's task is twofold: to outline the most appropriate preoperative and intraoperative medical treatment, and to direct the judicious use of additional diagnostic testing. We recommend beginning with the patient's current clinical status. Patients with decompensated heart failure should have surgery postponed in all but the most emergent circumstances. In cases of newly diagnosed heart failure, a cause should be determined through a diagnostic evaluation that is beyond the scope of this article. In those patients with chronic heart failure and an acute decompensation, one needs to ascertain the cause of the decompensation (that is, medical noncompliance, worsening ischaemia, atrial fibrillation, etc). Once the patient has stabilised, surgery should be postponed at least two weeks to ensure that the new medical regimen is appropriate and that the underlying process has been adequately addressed.

\section{Compensated heart failure}

Patients with compensated heart failure generally pose less of a challenge. If they have been clinically stable over the past several weeks, there is no indication for further testing. Many physicians advocate routine preoperative assessments of left ventricular ejection fraction (LVEF); however, there are little data to support this practice. Several studies have 


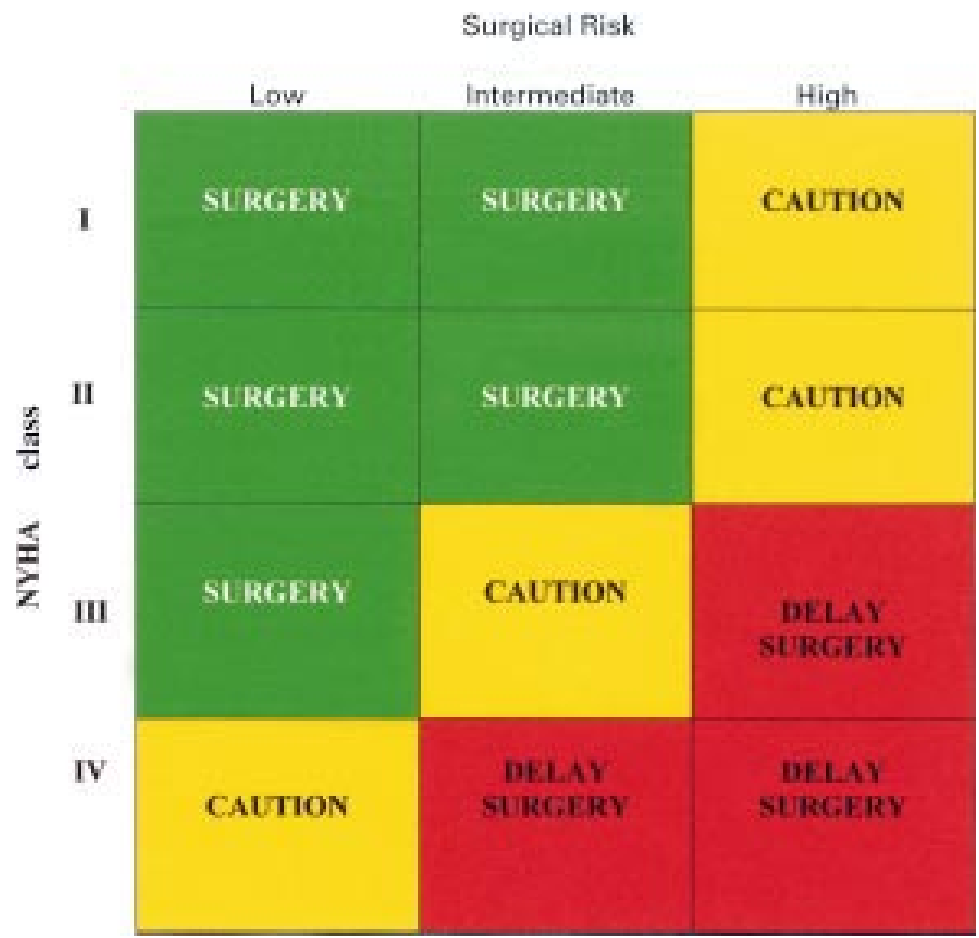

Figure 1 Risk stratification grid based on NYHA functional class (see box), and overall risk of surgery as defined in the text. Patients who fall into the "surgery" category may generally proceed with minimal risk. For those patients who fall into the "caution" category, we recommend haemodynamic monitoring with a pulmonary artery catheter. In addition, there should be case-by-case consideration for circulatory support with an intra-aortic balloon pump in decompensated patients undergoing an emergency, high risk procedure.

demonstrated a correlation between ejection fraction, particularly those less than $35 \%$, and adverse perioperative events; however, there are no data to suggest that patients with established heart failure benefit from routine repeat preoperative assessment of $\mathrm{LVEF}^{5-7}$ In fact, patients with heart failure and preserved LVEF (so called "diastolic dysfunction") do not tolerate tachycardia and intravascular volume shifts well, and may pose more perioperative management problems than stable, well compensated patients with a low LVEF.

In terms of optimising medical treatment, overwhelming evidence now points to the benefits of $\beta$ blockers and angiotensin converting enzyme (ACE) inhibitors in heart failure patients. ${ }^{8-12}$ Additionally, there have been reports of improved surgical outcomes with the use of perioperative $\beta$ blockers; however, the actual number of patients studied was quite small. ${ }^{13}{ }^{14}$ It should be emphasised that despite recent data suggesting a benefit of $\beta$ blocker treatment in New York Heart Association (NYHA) functional class III and IV heart failure, ${ }^{15}$ we do not recommend instituting these drugs in an acutely decompensated patient, particularly when emergency surgery is planned. Our goal is to deliver the surgeons a well compensated patient on an ACE inhibitor and $\beta$ blocker, with the addition of a diuretic and/or digoxin when indicated.

Accurate assessment of the patient's clinical status and the risk of the proposed surgery are fundamental components of the preoperative evaluation and are essential in making recommendations for intraoperative monitoring. We have developed a simple grid (fig 1) that assists
New York Heart Association functional class

Class I: Patient has no limitation of regular physical activities

Class II: Mild limitation of physical activities; comfortable at rest; normal physical activity results in dyspnoea, fatigue or angina

Class III: Major limitation of physical activities; comfortable at rest; minimal physical activity results in dyspnoea, fatigue, or angina

Class IV: Inability to perform any physical activity without symptoms; symptoms are present at rest, and are worsened with any activity

in this assessment by combining the risk of the proposed surgery with the patient's current functional status based on the NYHA classification (see box). Despite numerous techniques for evaluating cardiac status, the NYHA functional class has stood the test of time as an inexpensive, reproducible, and reliable indicator of long term prognosis. Low risk surgeries are defined as superficial procedures such as breast biopsies, endoscopic surgeries, or arthroscopic procedures. Intermediate risk procedures include orthopaedic, head and neck, genitourinary, and intra-abdominal procedures. High risk surgery includes major emergency procedures, aortic or vascular reconstructive surgery, and any prolonged procedure with predictable substantial changes in intravascular volume.

As shown in fig 1, we recommend delaying high risk surgery, when possible, in those patients who are in NYHA functional class III and IV. Additionally, we advocate intraoperative invasive haemodynamic monitoring with a pulmonary artery catheter in those patients who fall into the "caution" category. Admittedly, there are no conclusive data from randomised prospective trials to support this recommendation; however, it is in keeping with the American Society of Anesthesiologists class I and class II indications for the use of intraoperative pulmonary artery catheters. ${ }^{16}$ Furthermore, in those patients with decompensated heart failure requiring high risk, emergent surgery, we advise case-by-case consideration for circulatory support with an intra-aortic balloon pump.

In summary, accurate assessment of a patient's clinical status and the risk of the proposed surgery are critical elements in the preoperative evaluation of heart failure patients. Once the initial evaluation is complete, surgical risk can be minimised through a combination of optimal perioperative medical therapy with appropriate monitoring and support for higher risk cases.

1 Goldman L, Caldera DL, Nussbaum SR, et al. Multifactorial index of cardiac risk in noncardiac surgical procedures. N Engl f Med 1997;297:845-50.

2 Detsky AS, Abrams HB, McLaughlin JR, et al. Predicting 2 Detsky AS, Abrams HB, McLaughlin JR, et al. Predicting
cardiac complications in patients undergoing non-cardiac surgery. F Gen Intern Med 1986;1:211-19. 
3 Shah KB, Kleinman BS, Rao TLK, et al. Angina and other risk factors in patients with cardiac diseases undergoing

4 Ali MJ, Davison P, Pickett W, et al. ACC/AHA guidelines as predictors of postoperative cardiac outcomes. Can $\mathcal{F}$ Anesth

5 Pedersen T, Kelbaek H, Munck O. Cardiopulmonary complications in high-risk surgical patients: the value of preoperative radionuclide cardiography. Acta Anaesthesiol Scand 1990;34:183-9.

6 Pasternack PF, Imparato AM, Bear G, et al. The value of radionuclide angiography as a predictor of perioperative myocardial infarction in patients undergoing abdominal aortic aneurysm resection. $\mathcal{F}$ Vasc Surg. 1984;1:320-5.

7 Eagle KA, Brundage BH, Chaitman BR, et al. Guidelines for perioperative cardiovascular evaluation for noncardiac perioperative cardiovascular evaluation for noncardiac surgery: report of the American College of Cardiology/ American Heart Association task force on practical guidelines (committee on perioperative cardiovascular evaluation for

8 The CONSENSUS Trial Study Group. Effects of enalapril on mortality in severe congestive heart failure: results of the cooperative north Scandinavian enalapril survival study (CONSENSUS). N Engl f Med. 1987;316:1429-35.

9 The SOLVD Investigators. Effect of enalapril on survival in patients with reduced left ventricular ejection fractions and congestive heart failure. N Engl f Med. 1991;325:293-302.
10 Pfeffer MA, Braunwald E, Moye LA, et al on behalf of the SAVE investigators. Effect of captopril on mortality and morbidity in patients with left ventricular dysfunction after
myocardial infarction: survival and ventricular enlargement myocardial infarction: survival and ventric

11 CIBIS-II Investigators and Committees. The cardiac insufficiency bisoprolol study II (CIBIS-II): a randomized trial. Lancet. 1999;353:9-13.

12 MERIT-HF Investigators. Effect of metoprolol CR/XL in chronic heart failure: metoprolol randomised intervention trial in congestive heart failure (MERIT-HF). Lancet 1999; 353:2001-2007.

13 Poldermans D, Boersma E, Jeroen J, et al. The effect of bisoprolol on perioperative mortality and myocardial infarction in high-risk patients undergoing vascular surgery. $\mathrm{N} \mathrm{Engl} \mathcal{F}$ Med 1999;341:1789-94.

14 Pasternack PF, Imparato AM, Baumann FG, et al. The hemodynamics of $\beta$-blockade in patients undergoing abdominal aortic aneurysm repair. Circulation. 1987; 76(suppl 3, pt 2): III-1-7.

15 Witte K, Thackray S, Clarke AL, et al. Clinical trials update: IMPROVEMENT-HF, COPERNICUS, MUSTIC, ASPECT-II, APRICOT and HEART. Eur 7 Heart Failure ASPECT-II, APRI

16 American Society of Anesthesiologists. Practice guidelines for pulmonary artery catheterization: a report by the American Society of Anesthesiologists task force on pulmonary artery catheterization. Anesthesiology 1993;78:380-94.

\section{IMAGES IN CARDIOLOGY}

\section{Metastatic cardiac squamous cell carcinoma arising in the left ventricle}

A 71 year old man was diagnosed with lingual squamous cell carcinoma (T2 N0 M0) and was subjected to partial lingualectomy in June, 1997. Ten months after the operation, local signs of recurrences, such as ulceration in the oral cavity and multiple metastatic lesions, were found. Surprisingly, an ECG showed ST elevation in I, aVL, and V5-6, and ST depression in II, III, aVF, and V1-3 without any symptoms. Transthoracic echocardiography revealed multiple high echoic masses only in the left ventricular posterior wall and left ventricular lateral wall (top right, $\mathrm{LV}$, left ventricle, LA, left atrium, Ao, aorta). Although he received radiotherapy and chemotherapy, the patient died in March 1998. Pathological dissection of the heart revealed a cardiac tumour that occupied mainly the posterior and lateral walls of the left ventricle and almost extended over the left ventricle (bottom right). Interestingly, the cardiac tumour corresponded with the ST changes. Microscopic examination of haematoxylin and eosin stained tissue preparations revealed that the cardiac tumour was a squamous cell carcinoma, suggesting that it was a metastasis of the lingual cancer. Other metastases were found in the right lung, right and left adrenal glands, right and left kidney, right upper arm, and right forearm.

Almost all metastatic cardiac tumours have been reported to arise in the right side of the heart. There have been few reports about electrocardiographic changes induced by metastatic cardiac tumours. In this patient, the metastatic tumour occupied a large part of the left ventricular wall, which explains the ECG
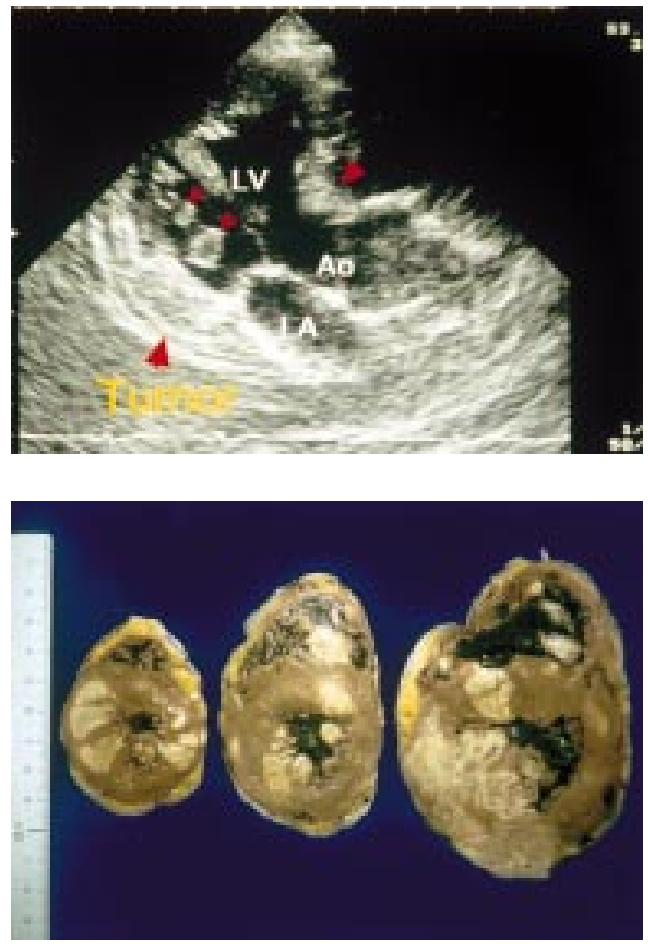

findings. This case indicates that a left ventricular mass accompanied with ST changes might indicate the presence of a metastatic tumour.

MASAKI SHIMOYAMA

FUMIYO KITAMURA-SASAKA CHIAKI SHIGEMASA masaki-tky@umin.ac.jp 